\title{
Response of the oxygen uptake efficiency slope to exercise training in patients with chronic kidney disease
}

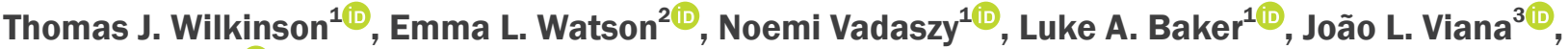 \\ Alice C. Smith ${ }^{1}$ \\ 'Leicester Kidney Lifestyle Team, Department of Health Science, University of Leicester, Leicester, UK \\ ${ }^{2}$ Department of Cardiovascular Sciences, University of Leicester, Leicester, UK \\ ${ }^{3}$ Research Center in Sports Sciences, Health Sciences and Human Development, CIDESD, University Institute of Maia, ISMAI, Maia, Portugal
}

Background: Chronic kidney disease (CKD) patients have poor cardiorespiratory fitness. Although cardiopulmonary exercise testing (CPET) is a universal assessment of cardiorespiratory fitness, values taken at 'peak' effort are strongly influenced by motivation and the choice of test endpoint. The oxygen uptake efficiency slope (OUES) integrates cardiovascular, musculoskeletal, and respiratory function into a single index to provide a more pragmatic and safer alternative to maximal testing. No research has explored whether exercise can improve the OUES in CKD patients.

Methods: Thirty-two patients with non-dialysis CKD were recruited into a 12-week exercise program consisting of mixed aerobic and resistance training three times a week. CPET was conducted at baseline, and then, following a 6 -week control period, at pre- and post-exercise intervention. Direct measurements of oxygen consumption $\left(\mathrm{V}_{2}\right)$ and ventilatory parameters were collected. The OUES was calculated as the relationship between $\mathrm{VO}_{2}$ and the $\log _{10}$ of minute ventilation ( $\dot{V}$ E).

Results: No changes were observed in any variable during the control period, although modest increases in $\mathrm{VO}_{2 \text { peak }}$ were observed. No meaningful changes were observed as a result of exercise in any cardiorespiratory value obtained. The OUES calculated at $100 \%, 90 \%, 75 \%$, and $50 \%$ of exercise duration did not change significantly after 12 weeks of exercise training.

Conclusion: Our results show that 12 weeks of exercise training had no beneficial effects on the OUES, which supports the modest change observed in $\mathrm{V}_{2 \text { peak }}$. The lack of change in the OUES and other parameters could indicate a dysfunctional cardiorespiratory response to exercise in patients with CKD, likely mediated by dysfunctional peripheral metabolic mechanisms.

Keywords: Chronic kidney disease, Exercise test, Exercise therapy, Oxygen consumption, Physical fitness

Received February 20, 2020; Revised March 27, 2020; Accepted May 2, 2020

Editor: Byung Ha Chung, The Catholic University of Korea, Seoul, Republic of Korea

Correspondence: Thomas J Wilkinson

Leicester Kidney Lifestyle Team, Department of Health Sciences, University of Leicester and University Hospitals of Leicester, Leicester, LE17RH, UK. E-mail: t.j.wilkinson@le.ac.uk

Copyright (C 2020 by The Korean Society of Nephrology

(a) This is an open-access article distributed under the terms of the Creative Commons Attribution Non-Commercial License (http://creativecommons.org/ licenses/by-nc-nd/4.0/), which permits unrestricted non-commercial use, distribution, and reproduction in any medium, provided the original work is properly cited. 


\section{Introduction}

Chronic kidney disease (CKD) is a global health problem affecting $8 \%$ to $16 \%$ of adults [1]. Defined as decreased kidney function for $>3$ months, individuals with CKD are at increased risk of morbidity and early mortality [1]. Cardiorespiratory fitness is defined as the integrated ability of the circulatory and respiratory systems to supply oxygen during sustained physical activity [2], and it is widely considered to be the best reflection of whole-body health and function [3]. Low cardiorespiratory fitness is strongly associated with early all-cause and cardiovascular-related mortality $[3,4]$ and presents a higher risk of early death than other common risk factors such as diabetes or hypertension [5]. Compared to the general population, CKD patients demonstrate a large reduction in cardiorespiratory fitness that occurs early in the disease process and declines concomitantly with renal function [6-8]. In patients with CKD, low cardiorespiratory fitness is associated with increased aortic stiffness and left ventricle afterload, poor left ventricle function, and a higher burden of cardiovascular risk [8], and it is unsurprisingly an independent predictor of early mortality $[9,10]$. Many patients with renal disease have cardiorespiratory values comparable to those with mild heart failure [11].

Cardiopulmonary exercise testing (CPET) is a universal assessment of cardiorespiratory fitness that uses a breath-by-breath gas exchange analysis to provide an objective and accurate measurement of oxygen uptake [3]. Although the measurement of maximal oxygen uptake $\left(\mathrm{V}_{2 \max }\right)$ is the most reliable measure of exercise capacity, this value is seldom achieved because it demands maximal effort from the individual being tested and a plateau in oxygen uptake despite an increasing exercise workload, which limits its usefulness in groups such as the elderly and those with chronic disease [12]. Consequently, in such groups and in patients with renal disease, cardiorespiratory fitness is typically derived from $\mathrm{VO}_{2 \text { peak }}$ because many patients prematurely stop the test due to fatigue or breathlessness [6]. $\mathrm{VO}_{2 \text { peak }}$ is also limited by the strong influence of motivation and the subjective choice of a test endpoint [12].

Baba et al [13] developed an objective, reproducible estimation of cardiorespiratory function called the oxygen uptake efficiency slope (OUES) that does not require a maximal exercise effort and might therefore be useful in populations, such as CKD patients, deemed high risk for maximal exercise testing [7]. The OUES integrates cardiovascular, musculoskeletal, and respiratory function into a single index [14]. Remaining relatively stable across CPET duration, the OUES represents the relationship (and efficiency) between oxygen uptake and total ventilation and provides an indication of cardiorespiratory reserve, so it could be a more pragmatic and safer alternative to maximal exercise testing in high-risk patients [7]. In patients with heart failure, the OUES has superior independent prognostic value over other commonly reported CPETderived values such as $\mathrm{VO}_{2 \text { peak }}$ and the $\mathrm{VE} / \mathrm{VCO}_{2}$ slope [15]. Although research on the subject is scarce, Kirkman et al [7] showed a $16 \%$ reduction in the OUES in CKD patients compared to healthy controls.

Generally, although not always $[16,17]$, exercise training can increase $\mathrm{VO}_{2 \text { peak }}$ in patients with $\mathrm{CKD}[17,18]$. However, little is known about the effect of exercise interventions on the OUES in any population. In patients with chronic heart failure (CHF) $[14,19]$ or coronary artery disease (CAD) $[20,21]$ and those undergoing hemodialysis (HD) [22], the OUES increased by $17 \%$ to $21 \%$ following exercise. An increase in the OUES suggests that a given oxygen uptake is achieved with lower ventilatory cost [21], although not all research supports those findings [23].

In this secondary analysis of previous research [16], we explore the response of the OUES to a 12-week exercise training program in patients with CKD. We hypothesized that 12 weeks of exercise would increase the OUES without necessarily changing the $\mathrm{VO}_{2 \text { peak }}$, indicating improvements in oxygen utilization efficiency.

\section{Methods}

\section{Participants}

Patients were recruited from nephrology outpatient clinics and included if they had CKD $3 b-5$ not requiring dialysis. Exclusion criteria were: 1 ) aged $<18$ years; 2) body mass index $>40 \mathrm{~kg} / \mathrm{m}^{2}$; 3) physical impairment preventing the completion of the study assessments; 4) myocardial infarction within $<6$ months; 5 ) any unstable chronic condition (e.g., diabetes); and 6) inability to give informed consent.

All procedures performed in studies involving human participants were in accordance with the ethical stan- 
dards of the institutional and/or national research committee at which the studies were conducted (National Research Ethics Committee, East Midlands-Northampton (13/EM/0344)) and with the 1964 Helsinki declaration and its later amendments or comparable ethical standards.

\section{Study design}

This is a secondary analysis of data collected during an exercise trial (registered at ISRCTN 36489137) that took place at the University Hospitals of Leicester NHS Trust between December 2013 and October 2016. A full description of the methods can be found in Watson et al [16]. Informed consent was obtained from all individual participants included in the study. Assessment outcomes were conducted at baseline and then again pre- and post-exercise intervention. To negotiate the absence of a control group, patients underwent a 6 -week control period prior to randomization between the baseline and pre-exercise assessment sessions.

\section{Exercise intervention}

Patients attended the research gym three times a week for 12 weeks. Patients were randomized (stratified for estimated glomerular filtration rate [eGFR]) into one of two supervised exercise groups:

Aerobic exercise group (AE group): $70 \%$ to $80 \%$ of maximum heart rate $[\mathrm{HR}], 30$ minutes duration in each session, performed on standard cardiovascular equipment (e.g. treadmill);

Combined exercise group (CE group): a combination of aerobic exercise (as above but for 20 minutes duration on two sessions, 30 minutes aerobic exercise only for the remaining session), plus resistance training ( $\sim 70 \%$ one repetition maximum, three sets of 8 to 12 repetitions) on a leg extension and leg press machine in the two sessions with 20 minutes of aerobic exercise.

\section{Cardiopulmonary exercise testing}

A symptom-limited, graded, maximal effort CPET was performed on a cycle ergometer (Lode Excalibur, Groningen, The Netherlands). Following a 3-minute warm-up at a resistance of 50 watts (W), patients were instructed to cycle $>60$ revolutions per minute (RPM). The work rate was increased by $1 \mathrm{~W}$ every 4 seconds $(15 \mathrm{~W} / \mathrm{min})$ using a ramp protocol of incremental workloads (Cortex MetaLyzer3B-R2 with Metasoft CPX software 3.0.68, Leipzig, Germany). The test was stopped if RPM dropped $<60$, the participant reached volitional exhaustion, or if the supervising cardiac nurse stopped the test because of echocardiogram changes or an undue rise in blood pressure. Total exercise duration was recorded in seconds.

Direct measurements of oxygen consumption $\left(\mathrm{VO}_{2}\right)$, minute ventilation (VE), and respiratory rate (RR) were obtained, and several derived variables such as the respiratory exchange ratio (RER, i.e., $\mathrm{V}_{2} / \dot{V} \mathrm{CO}_{2}$ ), ventilatory threshold (VT), and the ventilatory equivalents for oxygen $\left(\mathrm{VE} / \mathrm{VO}_{2}\right)$ and carbon dioxide $\left(\mathrm{VE} / \mathrm{VCO}_{2}\right)$ were calculated. The flowmeters and gas analyzers were calibrated before every test for accuracy and linearity with a syringe of known volume and precisely analyzed gas mixtures, respectively. The data pertaining to $\mathrm{VO}_{\text {peak }}$ following exercise were previously presented [16], but they are included here to provide a comparison with the utility of the OUES.

The OUES reflects the relationship between $\mathrm{VO}_{2}(\mathrm{~mL} /$ $\mathrm{min}$ ) and the $\log _{10}$ transformation of $\mathrm{VE}(\mathrm{L} / \mathrm{min})[13]$ :

$$
\dot{V} O_{2}=\left[a \log _{10} \dot{V} E\right]+b
$$

When $\mathrm{VO}_{2}$ is plotted on the y axis and $\log _{10} \dot{\mathrm{VE}}$ is plotted on the $x$ axis, the slope of this linear relationship, ' $a$ ', represents the OUES. An example of this relationship is shown in Fig. 1. Here, a steeper slope would represent more efficient oxygenation, and conversely, a shallower slope indicates that a greater amount of ventilation is required for any given oxygen uptake. To evaluate its usefulness as a cardiorespiratory index derived from a submaximal exercise test, the OUES was calculated from data taken at $50 \%, 75 \%, 90 \%$, and $100 \%$ of the total exercise duration (i.e., excluding the warm-up and recovery).

\section{Statistical analysis}

As a secondary per-protocol analysis [16], no prior sample size calculation is provided for the outcomes presented. Only patients with completed CPETs at each of the three study timepoints were included in this analysis. Data are presented as mean \pm standard deviation (SD), 


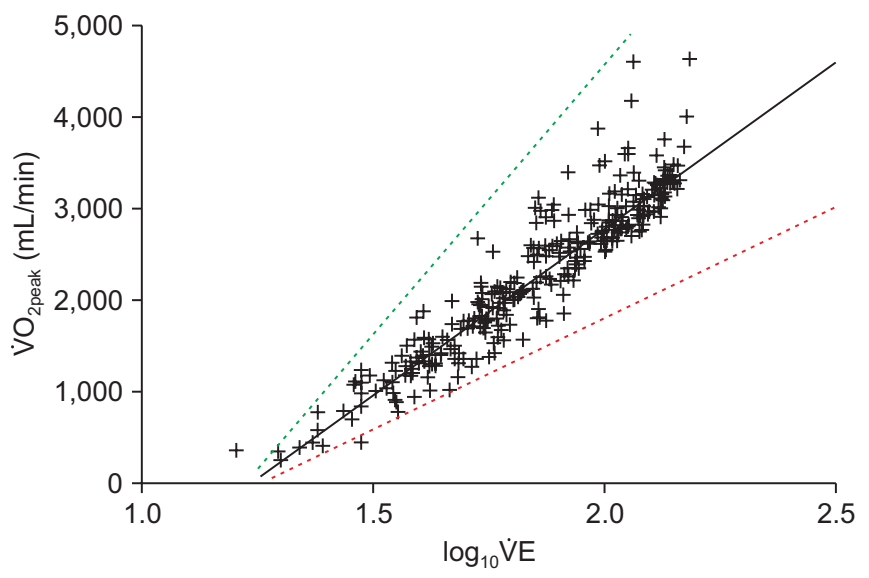

Figure 1. Example relationship between oxygen uptake $\left(\mathrm{VO}_{2}\right)$ and $\log _{10}$ transformed total ventilation (VंE) during incremental cardiopulmonary exercise testing in a 59-year-old male with chronic kidney disease (solid black line). The upper dashed line indicates a theoretically more efficient oxygen uptake as greater $\mathrm{V}_{2}$ is attained at a lower ventilatory cost. Conversely, the lower dashed line indicates a reduction in oxygen uptake efficiency as a greater amount of ventilation is required to attain any given $\mathrm{VO}_{2}$.

unless stated otherwise. The 95\% confidence intervals ( $95 \%$ CIs) were calculated for changes between the baseline, pre-, and post-exercise interventions. Within-group differences were assessed using paired-samples $t$ tests. Between-group differences were assessed using linear regression modeling adjusted for age, sex, ethnicity, eGFR, and baseline values. Differences in the OUES calculated at $50 \%, 75 \%, 90 \%$, and $100 \%$ of exercise duration at each timepoint were assessed using inter-item coefficients and Cronbach's $\alpha(r)$. Data were analyzed using IBM SPSS Statistics version 24.0 (IBM Corp., Armonk, NY, USA) and GraphPad PRISM 7.0 (San Diego, CA, USA). Significance was recognized as $P<0.05$.

\section{Results}

\section{Participant characteristics}

Basic patient demographics and clinical characteristics are shown in Table 1. The mean age was 61.1 \pm 12.1 and $56.3 \%$ of patients were female. Most patients were in CKD $3-4$ with an average eGFR of $25.3 \pm 7.4$. Clinical parameters were well controlled with a mean hemoglobin of $119.4 \pm$ $15.1 \mathrm{~g} / \mathrm{L}$ and blood pressure of $130.6 \pm 18.9 / 72.0 \pm 11.6$ $\mathrm{mmHg}$. No variable collected during the baseline CPET differed between those with CKD 3 and those with CKD 4/5,
Table 1. Basic patient demographics and clinical characteristics ( $n=32)$

\begin{tabular}{lc}
\hline \multicolumn{1}{c}{ Characteristic } & Value \\
\hline Age (yr) & $61.1 \pm 12.1$ \\
Sex (female) & $18(56.3)$ \\
Ethnicity & \\
White British & $22(68.8)$ \\
South Asian & $9(28.1)$ \\
Other & $1(3.1)$ \\
eGFR (mL/min) & \\
Stage 3b & $25.3 \pm 7.4$ \\
Stage 4 & $9(28.1)$ \\
Stage 5 & $21(65.6)$ \\
Etiology & $1(3.1)$ \\
Unknown & \\
lgA nephropathy & $18(56.3)$ \\
Diabetic nephropathy & $3(9.4)$ \\
PKD & $3(9.4)$ \\
Other & $2(6.3)$ \\
Body mass index (kg/m²) & $6(18.8)$ \\
Hemoglobin (g/L) & $30.1 \pm 6.0$ \\
Albumin (g/L) & $119.4 \pm 15.1$ \\
Hypertension & $40.8 \pm 2.9$ \\
Diabetes & $15(46.9)$ \\
Cardiovascular disease & $7(21.9)$ \\
Blood pressure (systolic/diastolic) (mmHg) & $4(12.5)$ \\
\hline & $18.9 / 72.0 \pm 11.6$ \\
\hline
\end{tabular}

Data are presented as mean \pm standard deviation or number (\%).

eGFR, estimated glomerular filtration rate; PKD, polycystic kidney disease.

${ }^{a}$ Data available for only 31 patients. Cardiovascular disease includes ischemic heart disease, stroke, and peripheral vascular disease.

including $\mathrm{VO}_{\text {peak }}\left(20.3 \pm 2.9\right.$ vs. $19.2 \pm 6.8 \mathrm{~mL} / \mathrm{min} \mathrm{kg}^{-1}, P=$ $0.513)$ and OUES (100\%) $(2,198.7 \pm 612.3$ vs. $2,169.3 \pm 717.0)$ (other data not shown).

\section{Changes during 6-week control period}

No significant changes in any variable were observed during the 6-week control period (Table 2). Although nonsignificant, modest increases in $\mathrm{VO}_{2 \text { peak }}$ peak $\dot{\mathrm{VE}} / \mathrm{VO}_{2}$, and peak RR were observed. Body mass was reduced by 0.7 (95\% CI, -1.5 to 0.1$) \mathrm{kg}$ following the 6 -week control period. The mean duration of the CPET differed by 1.0 (95\% CI, -42.4 to 40.5 ) second.

Response to 12 weeks of exercise in other parameters

A modest but nonsignificant mean increase of $0.8(95 \%$ 


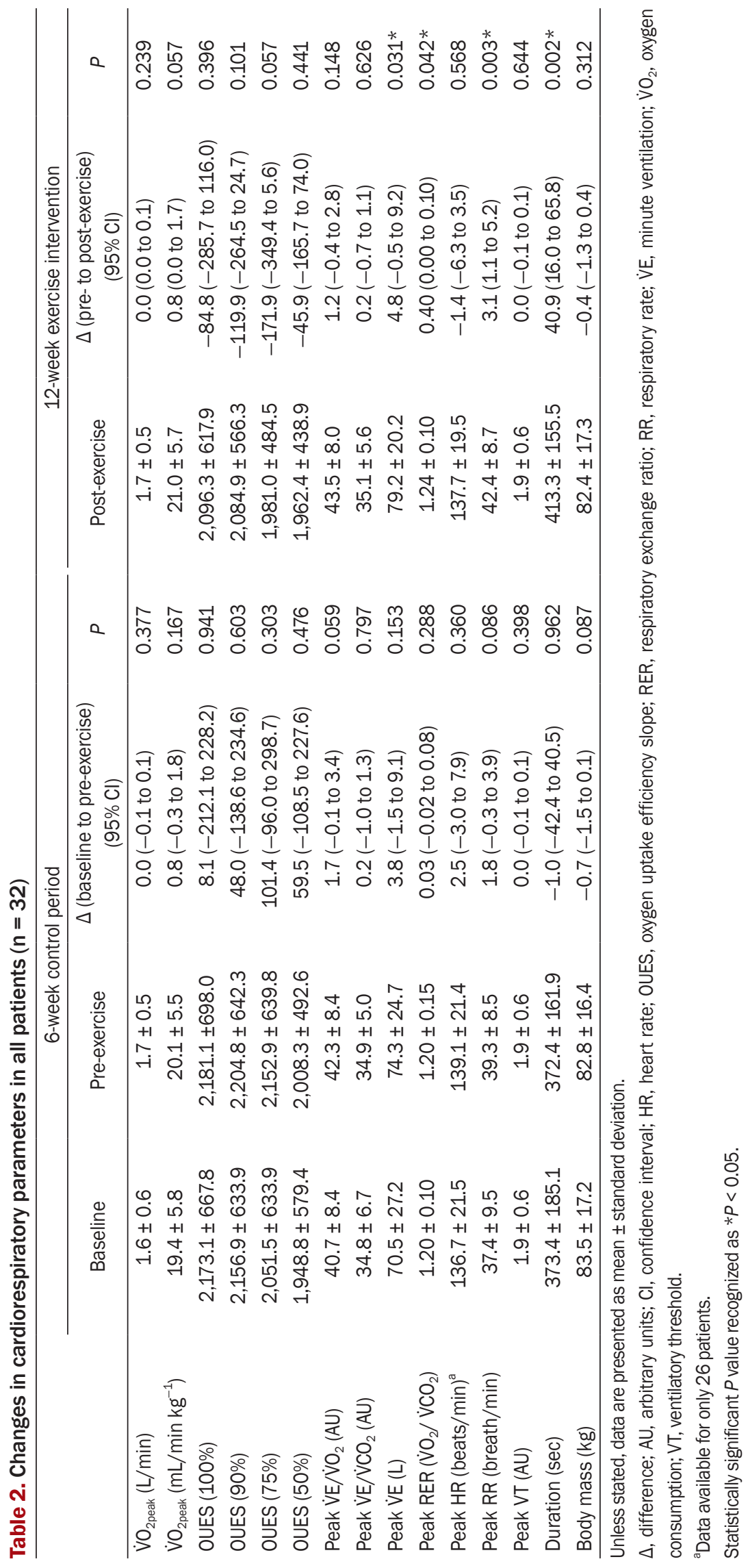


Table 3. Changes and differences in cardiorespiratory parameters in the two exercise groups

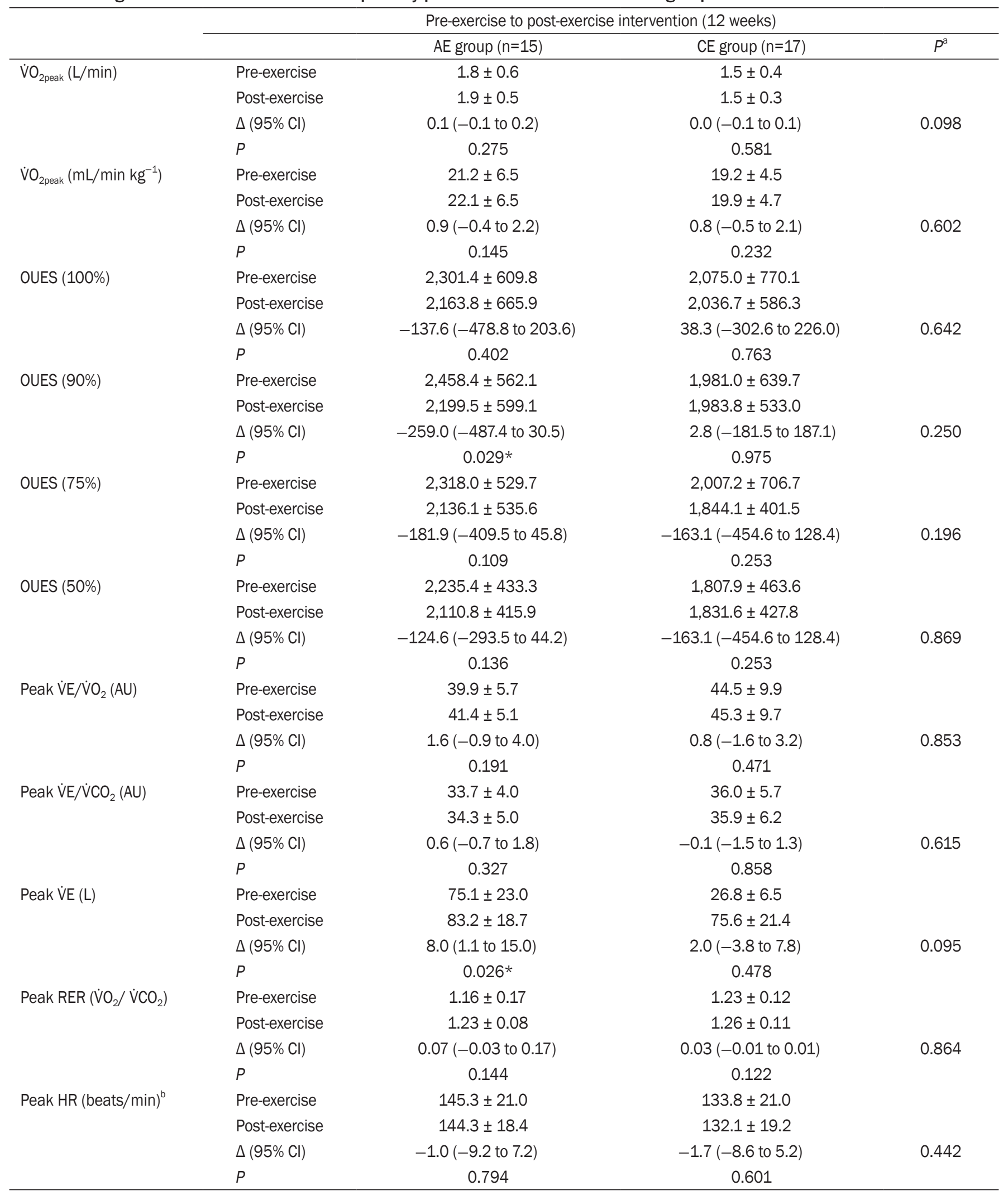


Table 3. Continued

\begin{tabular}{|c|c|c|c|c|}
\hline & \multicolumn{4}{|c|}{ Pre-exercise to post-exercise intervention (12 weeks) } \\
\hline & & AE group $(n=15)$ & CE group $(n=17)$ & $P^{\mathrm{a}}$ \\
\hline \multirow[t]{3}{*}{ Peak RR (breath/min) } & Pre-exercise & $39.5 \pm 9.1$ & $39.0 \pm 8.2$ & \\
\hline & $\Delta(95 \% \mathrm{Cl})$ & $3.5(-0.4$ to 7.3$)$ & $2.9(0.6$ to 5.2$)$ & 0.627 \\
\hline & $P$ & 0.073 & $0.017 *$ & \\
\hline \multirow{2}{*}{ Peak VT (AU) } & $\Delta(95 \% \mathrm{Cl})$ & $0.0(-0.18$ to 0.15$)$ & $0.0(-0.1$ to 0.1$)$ & 0.736 \\
\hline & $P$ & 0.873 & 0.593 & \\
\hline \multirow[t]{3}{*}{ Duration (sec) } & Pre-exercise & $412.5 \pm 193.1$ & $337.1 \pm 123.7$ & \\
\hline & Post-exercise & $457.4 \pm 165.9$ & $374.5 \pm 139.0$ & \\
\hline & $\Delta(95 \% \mathrm{Cl})$ & $44.9(6.2$ to 83.5$)$ & $37.4(1.1$ to 73.7$)$ & 0.153 \\
\hline
\end{tabular}

Unless stated, data are presented as mean \pm standard deviation.

$\Delta$, difference; $\mathrm{AE}$ group, aerobic exercise group; $\mathrm{AU}$, arbitrary units; $\mathrm{Cl}$, confidence interval; $\mathrm{CE}$ group, combined exercise group; HR, heart rate; OUES, oxygen uptake efficiency slope; RER, respiratory exchange ratio; $\mathrm{RR}$, respiratory rate; $\mathrm{V} E$, minute ventilation; $\dot{\mathrm{VO}}_{2}$, oxygen consumption; $\mathrm{VT}$, ventilatory threshold.

${ }^{a}$ Between-group $P$ value controlling for age, sex, ethnicity, eGFR, and pre-exercise value. ${ }^{b}$ Data available for only 26 patients (12 from AE group, 14 from CE group). Statistically significant $P$ value recognized as $* P<0.05$.

CI, 0.0 to 1.7$) \mathrm{mL} / \mathrm{min} \mathrm{kg}^{-1}(4 \%)$ was seen in $\mathrm{VO}_{2 \text { peak }}$ across all patients (Table 2), with no differences observed across groups $(P=0.602)$ (Table 3$)$. This increase was comparable to the 0.8 (95\% CI, -0.3 to 1.8 ) $\mathrm{mL} / \mathrm{min}^{-1}$ increase observed during the control period. No changes were observed in $\mathrm{VO}_{2 \text { peak }}$ expressed as $\mathrm{L} / \mathrm{min}(P=0.239)$.

No changes in breathing efficiency indices (peak $\mathrm{VE} / \mathrm{VO}_{2}$ and $\mathrm{VE} / \mathrm{VCO}_{2}$ ) were observed in either group, although VE increased by 8.0 (95\% CI, 1.1 to 15.0 ) liters in the $\mathrm{AE}$ group $(P=0.026)$. Peak RER increased when all patients were analyzed as a single group $(P=0.042)$, although no differences were observed between groups. Peak RR increased by 2.9 ( $95 \%$ CI, 0.6 to 5.2 ) breaths per minute in the CE group $(P=0.017)$. No change in peak HR was seen in either group $(P=0.568)$. The duration of the CPET increased following exercise training in both groups, by 44.9 and 37.4 seconds in the AE group $(P=0.026)$ and CE group $(P=0.044)$ groups, respectively (Table 2 and 3 ).

\section{Response to 12 weeks of exercise in the OUES}

The OUES calculated at $100 \%, 90 \%, 75 \%$, and $50 \%$ of exercise duration did not change after 12 weeks of exercise (Fig. 2 and Table 2), although we did observe a reduction in the OUES at $90 \%$ in the AE group $(P=0.029)$ (Table 3$)$. The responses to exercise produced more variable OUES

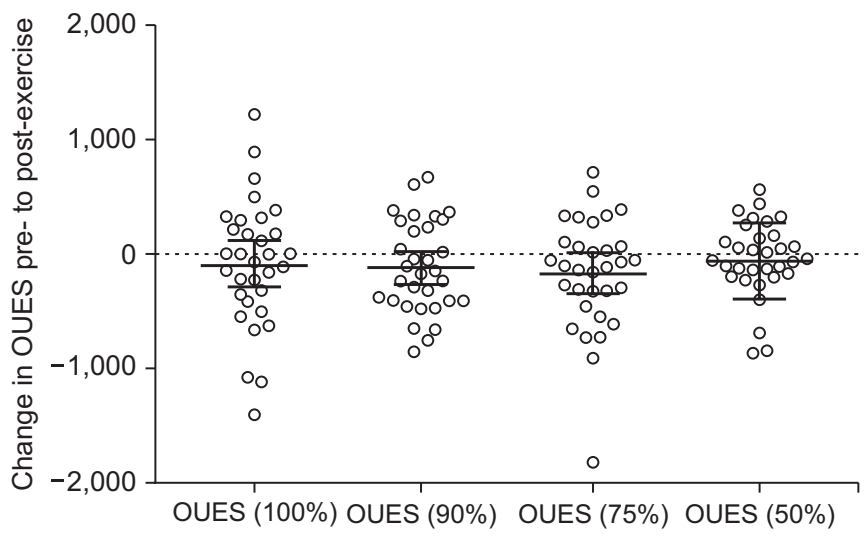

Figure 2. Mean changes in oxygen uptake efficiency slope (OUES) pre- to post-exercise calculated at $100 \%, 90 \%, 75 \%$, and $50 \%$ of exercise duration across all 32 patients. Dotted line represents no change.

values than $\mathrm{VO}_{2 \text { peak }}$ values. After training and across all patients, OUES ( $100 \%)$ decreased by $4 \%$ ( $95 \% \mathrm{CI},-13 \%$ to $5 \%)$, OUES ( $90 \%$ ) decreased by $5 \%$ ( $95 \% \mathrm{CI},-12 \%$ to $1 \%$ ), OUES ( $75 \%$ ) decreased by $8 \%$ ( $95 \% \mathrm{CI},-16 \%$ to $2 \%)$, and OUES (50\%) decreased by $2 \%(95 \% \mathrm{CI},-8 \%$ to $4 \%)$; this contrasts with a $4 \%(95 \% \mathrm{CI}, 0 \%$ to $8 \%)$ increase in $\mathrm{VO}_{2 \text { peak }}$. No correlation was found between the change in $\mathrm{VO}_{2 \text { peak }}$ and that in OUES $(100 \%)(\mathrm{r}=0.052, P=0.776)$. 
Table 4. Sensitivity and differences between OUES values calculated at different timepoints of exercise duration

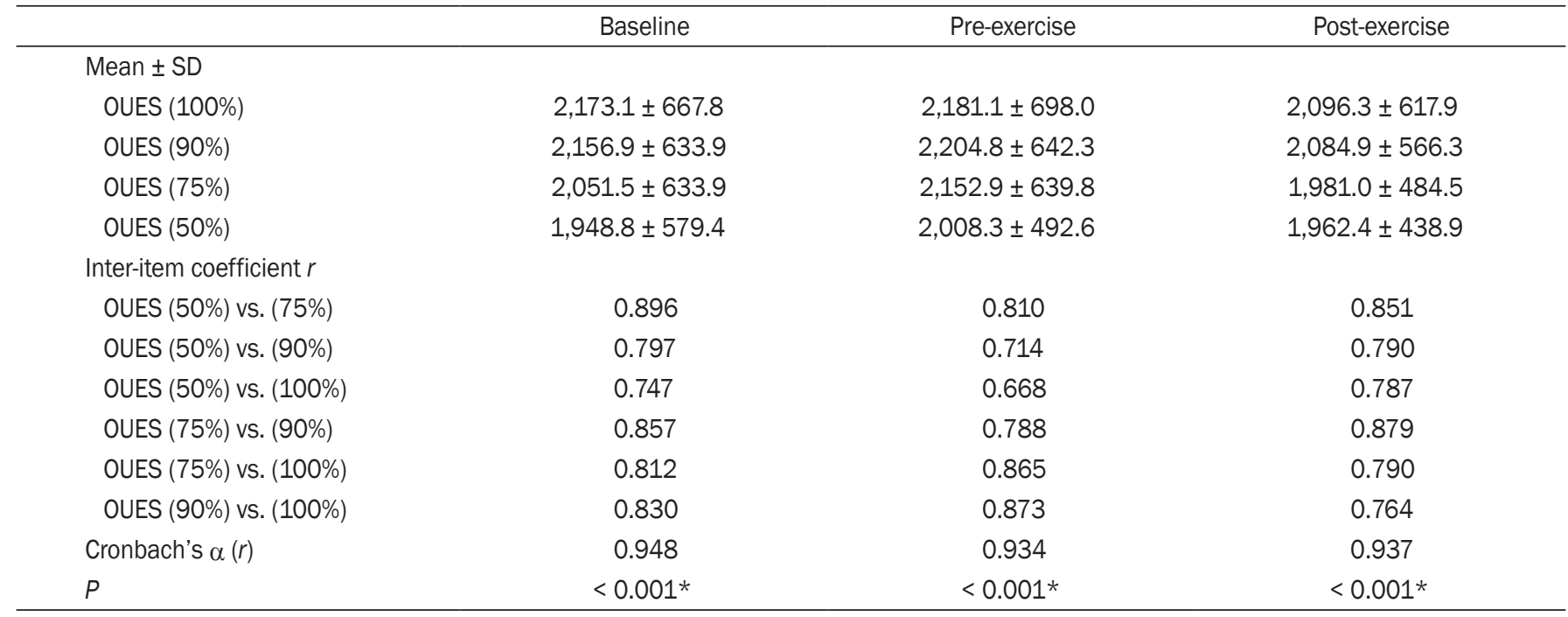

OUES, oxygen uptake efficiency slope; SD, standard deviation.

Differences in OUES calculated at 50\%,75\%,90\%, and 100\% of exercise duration at each timepoint were assessed using inter-item coefficients and Cronbach's $\alpha(r)$. Statistically significant $P$ value recognized as $* P<0.05$.
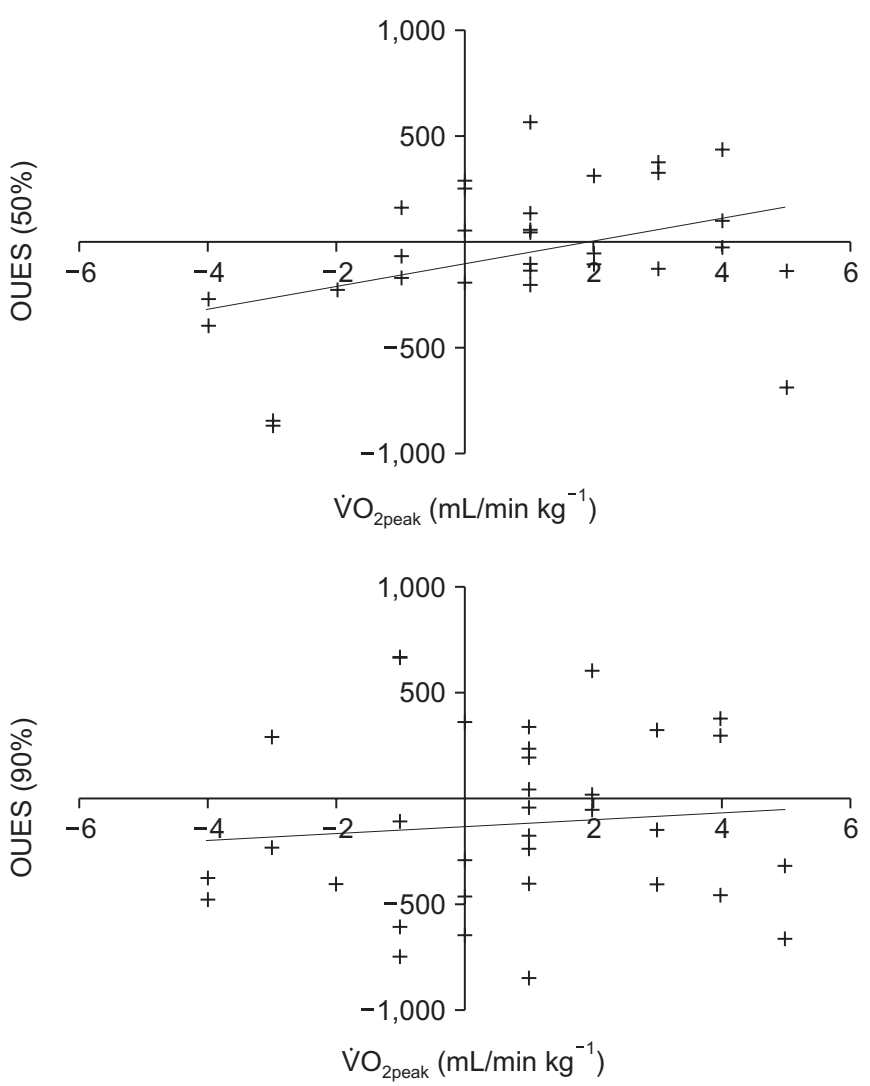

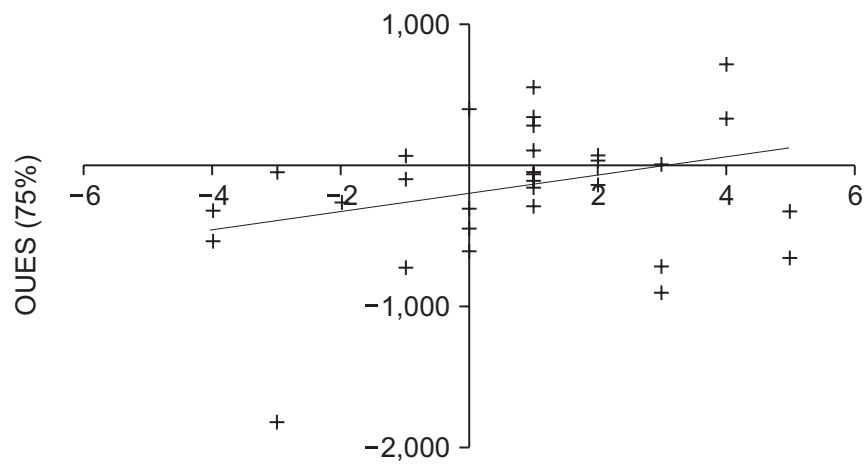

$\mathrm{VO}_{2 \text { peak }}\left(\mathrm{mL} / \mathrm{min} \mathrm{kg}^{-1}\right)$

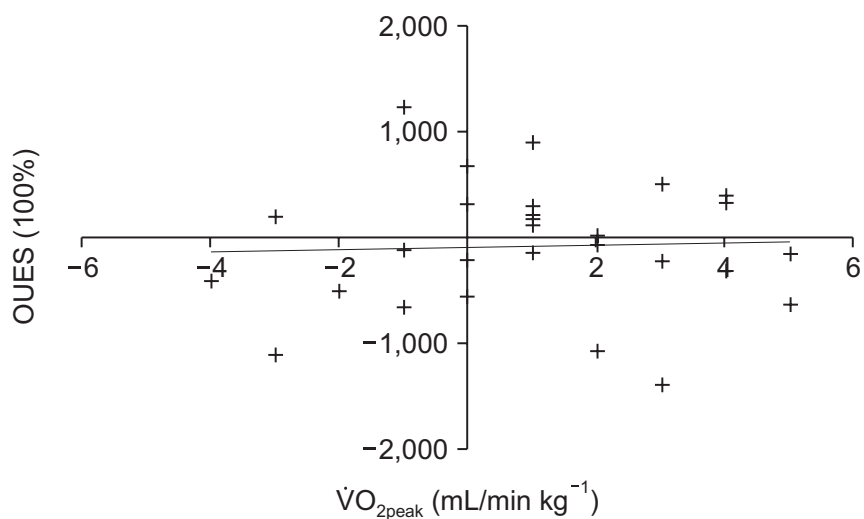

Figure 3. Association between changes pre- to post-exercise in oxygen uptake and oxygen uptake efficiency slope (OUES) calculated at $100 \%, 90 \%, 75 \%$, and $50 \%$ of exercise duration across all 32 patients.

$\dot{\mathrm{V}} \mathrm{O}_{2}$, oxygen consumption. 


\section{Sensitivity of submaximal OUES values}

The OUES values calculated at $50 \%, 75 \%$, and $90 \%$ of exercise duration were consistent with those from OUES (100\%) at each timepoint (Cronbach's $\alpha r=0.934$ to 0.948 ) (Table 4). OUES (90\%) was most highly correlated with OUES $100 \%$ at baseline (inter-item coefficient $r=0.830$ ) and pre-exercise $(r=0.873)$. The weakest association was observed between the OUES (50\%) values at different timepoints.

\section{Relationship between oxygen uptake and OUES values}

We observed significant associations between baseline $\mathrm{V}_{2 \text { peak }}$ and OUES $(100 \%)(\mathrm{r}=0.480, P=0.005)$, OUES (90\%) ( $\mathrm{r}=0.505, P=0.003)$, OUES (75\%) $(\mathrm{r}=0.640, P \leq$ $0.001)$, and OUES (50\%) $(\mathrm{r}=0.600, P \leq 0.001) . \mathrm{VO}_{2 \text { peak }}$ was also associated with VE $(r=0.743, P \leq 0.001)$. Fig. 3 shows the association between changes in the $\mathrm{VO}_{2 \text { peak }}$ and OUES values. The only significant association observed was with OUES (50\%) $(r=0.399, P=0.024)$.

\section{Discussion}

This is the first study to describe the effects of exercise on the OUES in patients with CKD. Our results show that 12 weeks of exercise had no beneficial effects on the OUES, and no meaningful differences appeared between the exercise modalities tested. The lack of change in the OUES supports the insignificant and modest change observed in $\mathrm{VO}_{2 \text { peak }}$. Exercise-induced responses in the OUES appeared to be more variable than changes in traditional parameters such as $\mathrm{VO}_{2 \text { peak }}$. The lack of change could indicate a dysfunctional cardiorespiratory response to exercise in patients with CKD.

Since the introduction of the OUES [13], its use as an objective, reproducible estimation of cardiorespiratory function in clinical populations has grown. Integrating cardiovascular, musculoskeletal, and respiratory function into a single index [14], the OUES represents the relationship between oxygen uptake and total ventilation. Given its linear presentation, the advantage of the OUES is its ability to obtain a valid measure with submaximal effort, which reduces reliance on parameters such as $\mathrm{V}_{2}$ peak that are strongly influenced by motivation and the choice of test endpoint [12]. Research exploring the role of the
OUES in kidney disease has been limited. Kirkman et al [7] found that the OUES of 31 patients was $16 \%$ lower than that of healthy controls. Suggestive of further underlying cardiovascular dysfunction, other parameters, such as $\dot{\mathrm{VO}}{ }_{2 \text { peak }}, \mathrm{VT}, \dot{\mathrm{VE}} / \mathrm{VCO}_{2}$, and $\mathrm{VE} / \mathrm{VO}_{2}$, were also impaired. Similarly, research by Tsuyuki et al [24] found a reduced OUES in HD patients. Given its prognostic value [15] and use as a potential marker of cardiorespiratory function, efforts to improve OUES values could be beneficial.

Given the lack of major differences between exercise modality groups, we focus on all patients as one cohort in this discussion. We observed a modest, nonsignificant increase in $\dot{\mathrm{VO}}_{2 \text { peak }}$ of $0.8 \mathrm{~mL} / \mathrm{min} \mathrm{kg}^{-1}$ or $\sim 4 \%$. This result is well below the generally accepted minimal clinically important difference of $1.5 \mathrm{~mL} / \mathrm{min} \mathrm{kg}^{-1}$ [25] and supports the conclusions of some previous exercise studies in CKD that showed inadequate change $[26,27]$. Nevertheless, it does contrast with most of the findings from a recent systematic review and meta-analysis that reported a pooled increase of 2.4 (1.0 to 3.8$) \mathrm{mL} / \mathrm{min} \mathrm{kg}^{-1}(\sim 11 \%)$ in patients with CKD following exercise [17]. Notably, we found that the increase after exercise was identical to that observed during the control period. Previous work by our group has shown that the minimal detectable change in $\mathrm{VO}_{2 \text { peak }}$ is $0.5 \mathrm{~mL} / \mathrm{min} \mathrm{kg}^{-1}$ [25], and as such, the change in $\mathrm{VO}_{2 \text { peak }}$ observed here is likely due to methodological limitations and the error inherent in this test. Improvements in relative $\mathrm{VO}_{2 \text { peak }}$ (i.e., adjusted for $\mathrm{kg}$ ) could also be explained by the concurrent changes in body mass seen throughout the study. That possibility is supported by the complete lack of change when $\mathrm{V}_{2 \text { peak }}$ is expressed in liters.

We expected the OUES to overcome the limitations associated with $\mathrm{VO}_{2 \text { peak }}$ measurement. However, contrary to our original hypothesis, we observed no changes in the OUES calculated at any stage of test duration in either group. This includes OUES (75\%), which was proposed [13] to replace $\mathrm{VO}_{2 \text { peak }}$ or OUES $(100 \%)$ because it does not rely on maximal effort. Our observations extend the results of Mourot et al [23], who reported that, despite an increase in $\mathrm{VO}_{2 \text { peak }}$, no change occurred in the OUES of healthy young women following 6 weeks of exercise. However, our results contrast with previous research in CHF $[14,19]$, CAD [20,21], and HD [22] that has shown improvements in the OUES in the region of $17 \%$ to $21 \%$, with changes in the OUES corresponding to improvements in $\mathrm{VO}_{2 \text { peak }}$. Interestingly, changes in the OUES cor- 
responded to changes in oxygen uptake but only during the first half of the exercise test. At the end of the test, changes in the OUES and $\mathrm{VO}_{2 \text { peak }}$ had no relationship. This finding could be due to the submaximal intensity of the first part of the test or variable attainments of true peak values in this group.

Many mechanisms can influence the OUES, including physiologic pulmonary dead space, which depends on the structural integrity of the lungs and the adequacy of pulmonary perfusion; the $\mathrm{P}_{\mathrm{a}} \mathrm{CO}_{2}$ set-point; and the timing and degree of metabolic acidosis, namely, the amount of blood perfusion to the working muscles $[12,19,22]$. Consequently, a large (superior) OUES depends on a substantial mass of working muscle, a vigorous and unimpaired blood flow to those muscles, efficient extraction and use of oxygen by those muscles, and the delayed appearance of lactic acidosis [12]. As such, improvements in the OUES in response to exercise are thought to be driven primarily by peripheral metabolic adaptations, such as increased aerobic metabolism [20], specifically increases in capillary density, muscle blood flow, and mitochondrial density that delay the onset of metabolic acidosis, thereby decreasing the ventilatory response during exercise [19-21].

The absence of change in the OUES of our CKD patients could be caused by a myriad of factors, although is likely due to dysfunctional peripheral metabolic mechanisms. Skeletal muscle mitochondrial abnormalities have been noted in patients with CKD [28]. To date, data from human studies are lacking, but skeletal muscle biopsies taken from CKD patients have revealed reduced mitochondrial density and mitochondrial DNA copy numbers, abnormal morphology, and reduced activity of pyruvate dehydrogenase [29], a key coupling enzyme in cellular respiration [30]. Dysfunctional mitochondria are an important source of reactive oxygen species, implicated in cardiovascular morbidity and mortality [31,32]. Patients with mitochondrial dysfunction are unable to adequately use $\mathrm{O}_{2}$ for oxidative phosphorylation; instead, lactic acid accumulates early in exercise, which leads to exaggerated circulatory and ventilatory responses [33]. Research by our group showed greater than normal deoxygenation of skeletal muscle hemoglobin in CKD patients during an incremental exercise test, which we hypothesized to indicate reduced mitochondrial and oxidative phosphorylation capacity [34]. We have also recently shown that at the cellular level, exercise training has no effect on mitochondrial mass or the mRNA expression of key proteins involved in mitochondrial biogenesis [35]. This points toward an intrinsic defect in our population in regard to their mitochondrial function.

Metabolic acidosis is a common complication in patients with CKD [36], and it has been implicated in the induction of mitochondrial dysfunction [36]. Early onset of lactate production during exercise testing has previously been observed in HD patients [37], and although research in earlier stages of CKD is limited, Sprick et al [38] found that metabolic acidosis contributes to exercise intolerance in CKD by promoting earlier fatigue and exacerbation of blood pressure reactivity during exercise. In deconditioned patients (such as those with chronic disease) who develop lactic acidosis early during exercise, the OUES will be expected to be diminished [12]. Exercise training has been shown to inhibit exercise-induced metabolic acidosis in healthy individuals and in those with CHF [22], but in the uremic milieu associated with CKD, this response could be diminished.

In our study, CKD-related peripheral limitations on exercise capacity are further indicated by an elevated ventilatory cost of oxygen uptake $\left(\mathrm{VE} / \mathrm{VO}_{2}\right)$ compared with values found in those without $\mathrm{CKD}$ [7]. In individuals with mitochondrial myopathies, steep $\mathrm{VE} / \mathrm{VO}_{2}$ slopes are observed because $\mathrm{O}_{2}$ delivery exceeds the capacity for utilization [38]. The ability of peripheral vessels to dilate in response to increased shear stress during exercise has emerged as an important determinant of exercise capacity. In patients with CKD, vascular endothelial dysfunction, as evidenced by increased arterial stiffness, is thought to contribute to exercise intolerance $[39,40]$. The lack of change in the OUES could also be caused by the highly variable response of this value to exercise, and it might not be a sensitive marker in CKD. This supports conclusions already drawn by others [23].

We observed little response to exercise in the other cardiorespiratory parameters we tested, although we did observe an increase in VEE and RR. The increase in VEE was found in the AE group only, and increased RR was identified in both groups. VE is a product of both tidal volume and RR [33]; therefore the increase seen in VE following exercise is likely partly explained by the increase in RR during the test. Following the intervention, the duration of the CPET increased, which might have increased 
the effort required to sustain the desired workload. This increased duration might also have required increased VE and RR. An increased peak VE (and/or RR) value with a relatively invariable $\mathrm{VO}_{2}$, as observed in our sample, could also be characteristic of inefficient breathing patterns [33]. Despite an increase in VE, we observed no change in the $\mathrm{VE} / \mathrm{V} \mathrm{CO}_{2}$ slope, suggesting no improvement in $\mathrm{CO}_{2}$ exhalation and no reduction in lactic acid production [19]. Kirkman et al [7] previously showed an increased $\mathrm{VE} / \mathrm{VCO}_{2}$ slope in patients with $\mathrm{CKD}$ versus healthy controls. The high $\mathrm{VE} / \mathrm{VCO}_{2}$ slope observed in our sample could represent impaired ventilatory efficiency, and our mean values at each timepoint, all $>34$, indicate an abnormal ventilatory response $[33,41]$ similar to those seen in patients with pulmonary disease and CHF [33].

Although it is limited by the absence of a non-exercising control group, our study benefits from having a control period in which we could assess normal variations in the parameters reported. This was important because it allowed us to observe increases in $\mathrm{VO}_{2 \text { peak }}$ comparable to those from the 12-week exercise program, supporting the finding that aerobic capacity was unchanged by exercise. It is unlikely that this was caused by an inadequate training stimulus (i.e., duration or intensity) because significant improvements in body composition, strength, and other physical functions were observed as a result of the program [16]. We were able to show good reproducibility of the OUES before and after this control period, with the OUES $(100 \%)$ differing by just $0.4 \%$. As a secondary analysis, the results here might not be powered to detect statistically significant changes, and therefore they need to be confirmed in larger trials with this population.

In conclusion, we were unable to obtain an improvement in the OUES after 12 weeks of exercise in patients with CKD. The OUES was hypothesized to be a useful index that circumvents the methodological limitations of maximal exercise testing in evaluating cardiorespiratory reserve in patients. However, although the heterogeneous variation in the OUES response could partly explain our findings, the lack of change in the OUES supports the limited change in $\mathrm{VO}_{2 \text { peak }}$ and other cardiorespiratory parameters measured. Thus, our findings could indicate a dysfunctional cardiorespiratory response to exercise in patients with CKD.

\section{Conflicts of interest}

The authors declare that no conflict of interest exists. The views expressed are those of the authors and not necessarily those of the National Health Service (NHS), the National Institute for Health Research (NIHR), or the Department of Health.

\section{Funding}

This research was part-funded by the Stoneygate Trust and supported by the National Institute for Health Research Leicester Biomedical Research Centre. At the time of writing this manuscript, Emma L Watson was supported by a Kidney Research UK Post-Doctoral Fellowship.

\section{Acknowledgments}

This report describes independent research supported by the National Institute for Health Research Leicester Biomedical Research Centre. The views expressed are those of the author(s) and not necessarily those of the NHS, the National Institute for Health Research Leicester BRC, or the Department of Health. The authors thank the participants for taking part in the study. We also thank other members of the Leicester Kidney Lifestyle Team for their help in acquiring some of the cardiopulmonary tests and supervising the exercise training.

\section{Authors' contributions}

Emma L. Watson, João L. Viana, and Alice C. Smith contributed to the original conception and design of the study. Thomas J. Wilkinson contributed to conception of the current retrospective analysis. All authors contributed to the acquisition, analysis, or interpretation of data. Thomas J. Wilkinson drafted the manuscript. All authors critically revised the manuscript, gave final approval, and agree to be accountable for all aspects of the work to ensure integrity and accuracy.

\section{References}

[1] Webster AC, Nagler EV, Morton RL, Masson P. Chronic kidney disease. Lancet 2017;389:1238-1252.

[2] Caspersen CJ, Powell KE, Christenson GM. Physical activ- 
ity, exercise, and physical fitness: definitions and distinctions for health-related research. Public Health Rep 1985; 100:126-131.

[3] Imboden MT, Harber MP, Whaley MH, Finch WH, Bishop DL, Kaminsky LA. Cardiorespiratory fitness and mortality in healthy men and women. J Am Coll Cardiol 2018;72: 2283-2292.

[4] Sieverdes JC, Sui X, Lee DC, et al. Physical activity, cardiorespiratory fitness and the incidence of type 2 diabetes in a prospective study of men. Br J Sports Med 2010;44:238-244.

[5] Blair SN, Sallis RE, Hutber A, Archer E. Exercise therapythe public health message. Scand J Med Sci Sports 2012;22: e24-e28.

[6] Zelle DM, Klaassen G, van Adrichem E, Bakker SJ, Corpeleijn E, Navis G. Physical inactivity: a risk factor and target for intervention in renal care. Nat Rev Nephrol 2017;13:318.

[7] Kirkman DL, Muth BJ, Stock JM, Townsend RR, Edwards DG. Cardiopulmonary exercise testing reveals subclinical abnormalities in chronic kidney disease. Eur J Prev Cardiol 2018;25:1717-1724.

[8] Howden EJ, Weston K, Leano R, et al. Cardiorespiratory fitness and cardiovascular burden in chronic kidney disease. J Sci Med Sport 2015;18:492-497.

[9] Gulati M, Black HR, Arnsdorf MF, Shaw LJ, Bakris GL. Kidney dysfunction, cardiorespiratory fitness, and the risk of death in women. $J$ Womens Health (Larchmt) 2012;21:917924.

[10] Ahmed A, Qureshi WT, Brawner CA, et al. Prognostic value of cardiorespiratory fitness in patients with chronic renal dysfunction: the FIT (Henry Ford Exercise Testing) project. Circulation 2015;132(suppl_3):A13614.

[11] Janicki JS, Weber KT, McElroy PA. Use of the cardiopulmonary exercise test to evaluate the patient with chronic heart failure. Eur Heart J 1988;9 Suppl H:55-58.

[12] Hollenberg M, Tager IB. Oxygen uptake efficiency slope: an index of exercise performance and cardiopulmonary reserve requiring only submaximal exercise. J Am Coll Cardiol 2000;36:194-201.

[13] Baba R, Nagashima M, Goto M, et al. Oxygen uptake efficiency slope: a new index of cardiorespiratory functional reserve derived from the relation between oxygen uptake and minute ventilation during incremental exercise. J Am Coll Cardiol 1996;28:1567-1572.

[14] Van Laethem C, Van De Veire N, De Backer G, et al. Response of the oxygen uptake efficiency slope to exercise training in patients with chronic heart failure. Eur J Heart
Fail 2007;9:625-629.

[15] Davies LC, Wensel R, Georgiadou P, et al. Enhanced prognostic value from cardiopulmonary exercise testing in chronic heart failure by non-linear analysis: oxygen uptake efficiency slope. Eur Heart J 2006;27:684-690.

[16] Watson EL, Gould DW, Wilkinson TJ, et al. Twelve-week combined resistance and aerobic training confers greater benefits than aerobic training alone in nondialysis CKD. Am J Physiol Renal Physiol 2018;314:F1188-F1196.

[17] Vanden Wyngaert K, Van Craenenbroeck AH, Van Biesen W, et al. The effects of aerobic exercise on eGFR, blood pressure and VO2peak in patients with chronic kidney disease stages 3-4: A systematic review and meta-analysis. PLoS One 2018;13:e203662.

[18] Heiwe S, Jacobson SH. Exercise training in adults with CKD: a systematic review and meta-analysis. Am J Kidney Dis 2014;64:383-393.

[19] Gademan MG, Swenne CA, Verwey HF, et al. Exercise training increases oxygen uptake efficiency slope in chronic heart failure. Eur J Cardiovasc Prev Rehabil 2008;15: 140-144.

[20] Prado DM, Rocco EA, Silva AG, et al. Effects of continuous vs interval exercise training on oxygen uptake efficiency slope in patients with coronary artery disease. Braz J Med Biol Res 2016;49:e4890.

[21] Defoor J, Schepers D, Reybrouck T, Fagard R, Vanhees L. Oxygen uptake efficiency slope in coronary artery disease: clinical use and response to training. Int J Sports Med 2006; 27:730-737.

[22] Tsuyuki K, Kimura Y, Chiashi K, et al. Oxygen uptake efficiency slope as monitoring tool for physical training in chronic hemodialysis patients. Ther Apher Dial 2003;7: 461-467.

[23] Mourot L, Perrey S, Tordi N, Rouillon JD. Evaluation of fitness level by the oxygen uptake efficiency slope after a short-term intermittent endurance training. Int $J$ Sports Med 2004;25:85-91.

[24] Tsuyuki K, Baba R, Matsushita C, et al. Minimum exercise intensity level required to detect reliable oxygen uptake efficiency slope in patients with chronic hemodialysis. Nihon Toseki Igakkai Zasshi 1999;32:271-277.

[25] Wilkinson TJ, Watson EL, Xenophontos S, Gould DW, Smith AC. The "minimum clinically important difference" in frequently reported objective physical function tests after a 12-week renal rehabilitation exercise intervention in Nondialysis chronic kidney disease. Am J Phys Med Rehabil 
2019;98:431-437.

[26] Baria F, Kamimura MA, Aoike DT, et al. Randomized controlled trial to evaluate the impact of aerobic exercise on visceral fat in overweight chronic kidney disease patients. Nephrol Dial Transplant 2014;29:857-864.

[27] Leehey DJ, Collins E, Kramer HJ, et al. Structured exercise in obese diabetic patients with chronic kidney disease: a randomized controlled trial. Am J Nephrol 2016;44:54-62.

[28] Yazdi PG, Moradi H, Yang JY, Wang PH, Vaziri ND. Skeletal muscle mitochondrial depletion and dysfunction in chronic kidney disease. Int J Clin Exp Med 2013;6:532-539.

[29] Gamboa JL, Billings FT 4th, Bojanowski MT, et al. Mitochondrial dysfunction and oxidative stress in patients with chronic kidney disease. Physiol Rep 2016;4:e12780.

[30] Xu C, Kasimumali A, Guo X, et al. Reduction of mitochondria and up regulation of pyruvate dehydrogenase kinase 4 of skeletal muscle in patients with chronic kidney disease. Nephrology (Carlton) 2020;25:230-238.

[31] López-Armada MJ, Riveiro-Naveira RR, Vaamonde-García C, Valcárcel-Ares MN. Mitochondrial dysfunction and the inflammatory response. Mitochondrion 2013;13:106-118.

[32] Dai L, Golembiewska E, Lindholm B, Stenvinkel P. Endstage renal disease, inflammation and cardiovascular outcomes. Contrib Nephrol 2017;191:32-43.

[33] Forman DE, Myers J, Lavie CJ, Guazzi M, Celli B, Arena R. Cardiopulmonary exercise testing: relevant but underused. Postgrad Med 2010;122:68-86.

[34] Wilkinson TJ, White AEM, Nixon DGD, Gould DW, Watson
EL, Smith AC. Characterising skeletal muscle haemoglobin saturation during exercise using near-infrared spectroscopy in chronic kidney disease. Clin Exp Nephrol 2019;23:32-42.

[35] Watson EL, Baker LA, Wilkinson TJ, et al. Reductions in skeletal muscle mitochondrial mass are not restored following exercise training in patients with chronic kidney disease. FASEB J 2020;34:1755-1767.

[36] Kraut JA, Kurtz I. Metabolic acidosis of CKD: diagnosis, clinical characteristics, and treatment. Am J Kidney Dis 2005;45:978-993.

[37] Painter P, Zimmerman SW. Exercise in end-stage renal disease. Am J Kidney Dis 1986;7:386-394.

[38] Sprick JD, Morison DL, Fonkoue IT, et al. Metabolic acidosis augments exercise pressor responses in chronic kidney disease. Am J Physiol Regul Integr Comp Physiol 2019;317: R312-R318.

[39] Taivassalo T, Jensen TD, Kennaway N, DiMauro S, Vissing J, Haller RG. The spectrum of exercise tolerance in mitochondrial myopathies: a study of 40 patients. Brain 2003;126(Pt 2):413-423.

[40] Van Craenenbroeck AH, Van Craenenbroeck EM, Van Ackeren $\mathrm{K}$, et al. Impaired vascular function contributes to exercise intolerance in chronic kidney disease. Nephrol Dial Transplant 2016;31:2064-2072.

[41] Corrà U, Mezzani A, Bosimini E, Giannuzzi P. Cardiopulmonary exercise testing and prognosis in chronic heart failure: a prognosticating algorithm for the individual patient. Chest 2004;126:942-950. 